\title{
HERMES Measurements of Collins and Sivers Asymmetries from a Transversely Polarised Hydrogen Target
}

\author{
Markus Diefenthaler (on behalf of the HERMES collaboration) \\ Friedrich-Alexander-Universität Erlangen-Nürnberg - Physikalisches Institut II \\ Erwin-Rommel-Straße 1, 91058 Erlangen - Germany
}

\begin{abstract}
Azimuthal single-spin asymmetries (SSA) in semi-inclusive electroproduction of $\pi$ mesons and charged $K$-mesons in deep-inelastic scattering of positrons and electrons on a transversely polarised hydrogen target were observed. Significant SSA amplitudes for both the Collins and the Sivers mechanism are presented for the full data set recorded with transverse target polarisation at the HERMES experiment.
\end{abstract}

\section{Contribution}

In 2005 the HERMES collaboration published first evidence for azimuthal single-spin asymmetries (SSA) in the semi-inclusive production of charged pions on a transversely polarised hydrogen target [2]. Significant signals for both the Collins [3] and Sivers mechanisms [4] were observed in data recorded during the 2002-2003 running period of the HERMES experiment. Below we present a preliminary analysis of these data combined with additional data taken in the years 2003-2005; i.e. an preliminary analysis of the full data set with transverse target polarisation [1]. All data were recorded at a beam energy of $27.6 \mathrm{GeV}$ using a transversely nuclear-polarised hydrogen-target internal to the HERA storage ring at DESY. The HERMES dual-radiator ring-imaging Čerenkov counter allows full $\pi^{ \pm}, K^{ \pm}, p$ separation for all particle momenta within the range $2 \mathrm{GeV}<\boldsymbol{P}_{\mathrm{h}}<15 \mathrm{GeV}$. Therefore, a preliminary analysis of SSA in the electroproduction of charged kaons on a transversely polarised target is also presented. In addition the measurement is accompanied by an preliminary analysis of reconstructed neutral-pion events.

At leading twist, the longitudinal momentum and spin of the quarks inside the nucleon are described by three parton distribution functions: the well-known momentum distribution $q\left(x, Q^{2}\right)$, the known helicity distribution $\Delta q\left(x, Q^{2}\right)[5]$ and the (experimentally) unknown transversity distribution $\delta q\left(x, Q^{2}\right)[6,7,8,9]$. In the helicity basis, transversity is related to a quark-nucleon forward scattering amplitude involving helicity flip of both nucleon and quark $\left(N^{\Rightarrow} q \leftarrow \rightarrow N^{\leftarrow} q \rightarrow\right)$. As it is chiral-odd, transversity cannot be probed in inclusive deep-inelastic scattering (DIS). At HERMES transversity in conjunction with the chiral-odd Collins fragmentation function [3] is accessible in SSA in semi-inclusive DIS on a transversely polarised target (Collins mechanism). The Collins fragmentation function describes the correlation between the transverse polarisation of the struck quark and the transverse momentum $\boldsymbol{P}_{\mathrm{h} \perp}$ of the hadron produced. As it is also odd under naive time reversal (T-odd) it can produce a SSA, i.e. a left-right asymmetry in the momentum distribution of the produced hadrons in the plane transverse to the virtual photon direction.

The Sivers mechanism can also cause a SSA: The T-odd Sivers distribution function [4] describes the correlation between the transverse polarisation of the nucleon and the transverse momentum $\boldsymbol{p}_{\perp}$ of the quarks within. A non-zero Sivers mechanism provides 

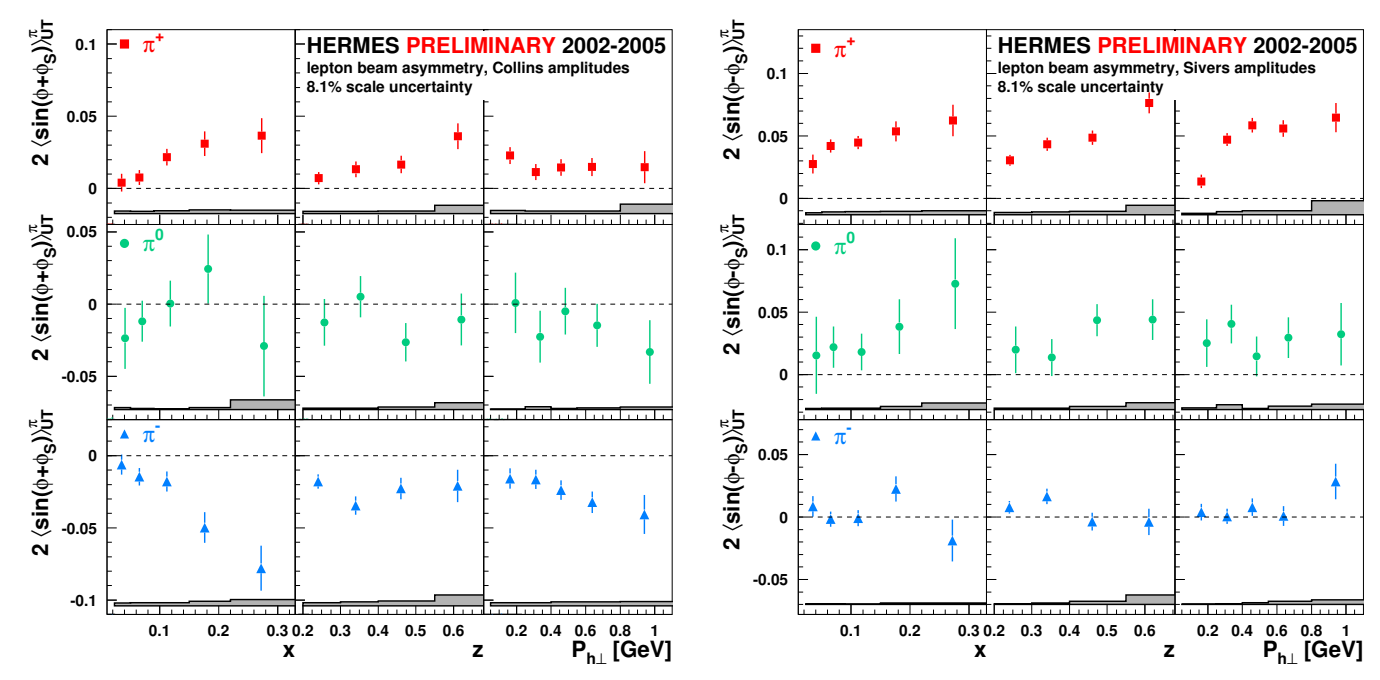

Figure 1: Collins amplitudes (left column) and Sivers amplitudes (right column) for $\pi$ mesons (as labelled) as function of $x, z$ and $\boldsymbol{P}_{\mathrm{h} \perp}$. The error bands represent the maximal systematic uncertainty; the common overall $8.1 \%$ scaling uncertainty is due to the target polarisation uncertainty.

a non-zero Compton amplitude involving nucleon helicity flip without quark helicity flip $\left(N^{\Rightarrow} q^{\leftarrow} \rightarrow N^{\Leftarrow} q^{\leftarrow}\right)$, which must therefore involve orbital angular momentum of the quark inside the nucleon $[10,11]$.

With a transversely polarised target, the azimuthal angle $\phi_{\mathrm{S}}$ of the target spin direction in the "介" state is observable in addition to the azimuthal angle $\phi$ of the detected hadron. Both azimuthal angles are defined about the virtual-photon direction with respect to the lepton scattering plane. The additional degree of freedom $\phi_{\mathrm{S}}$, not available with a longitudinally polarised target, results in distinctive signatures: $\sin \left(\phi+\phi_{\mathrm{S}}\right)$ for the Collins mechanisms and $\sin \left(\phi-\phi_{\mathrm{S}}\right)$ for the Sivers mechanism [12].

The corresponding azimuthal amplitudes azimuthal amplitudes are extracted using maximum likelihood fits. The Collins amplitudes $\left\langle\sin \left(\phi+\phi_{\mathrm{S}}\right)\right\rangle_{\mathrm{UT}}^{\mathrm{h}}$ and the Sivers mechanism $\left\langle\sin \left(\phi-\phi_{\mathrm{S}}\right)\right\rangle_{\mathrm{UT}}^{\mathrm{h}}$ were extracted simultaneously to avoid cross-contamination. To allow for contribution from all theoretically possible Fourier modulations the terms for $\sin \phi_{\mathrm{S}}$, $\sin \left(2 \phi-\phi_{\mathrm{S}}\right)$ and $\sin \left(3 \phi-\phi_{\mathrm{S}}\right)$ have to be added in the probability density function $\mathrm{F}$ :

$$
\begin{aligned}
\mathrm{F}\left(2\left\langle\sin \left(\phi \pm \phi_{S}\right)\right\rangle_{\mathrm{UT}}^{\mathrm{h}}, \ldots, \phi, \phi_{S}\right)=\frac{1}{2}\left(1+P_{\alpha}^{z}(\right. & 2\left\langle\sin \left(\phi+\phi_{S}\right)\right\rangle_{\mathrm{UT}}^{\mathrm{h}} \cdot \sin \left(\phi+\phi_{S}\right)+ \\
& 2\left\langle\sin \left(\phi-\phi_{S}\right)\right\rangle_{\mathrm{UT}}^{\mathrm{h}} \cdot \sin \left(\phi-\phi_{S}\right)+ \\
& 2\left\langle\sin \left(3 \phi-\phi_{S}\right)\right\rangle_{\mathrm{UT}}^{\mathrm{h}} \cdot \sin \left(3 \phi-\phi_{S}\right)+ \\
& 2\left\langle\sin \left(2 \phi-\phi_{S}\right)\right\rangle_{\mathrm{UT}}^{\mathrm{h}} \cdot \sin \left(2 \phi-\phi_{S}\right)+ \\
& \left.\left.2\left\langle\sin \phi_{S}\right\rangle_{\mathrm{UT}}^{\mathrm{h}} \cdot \sin \phi_{S}\right)\right)
\end{aligned}
$$



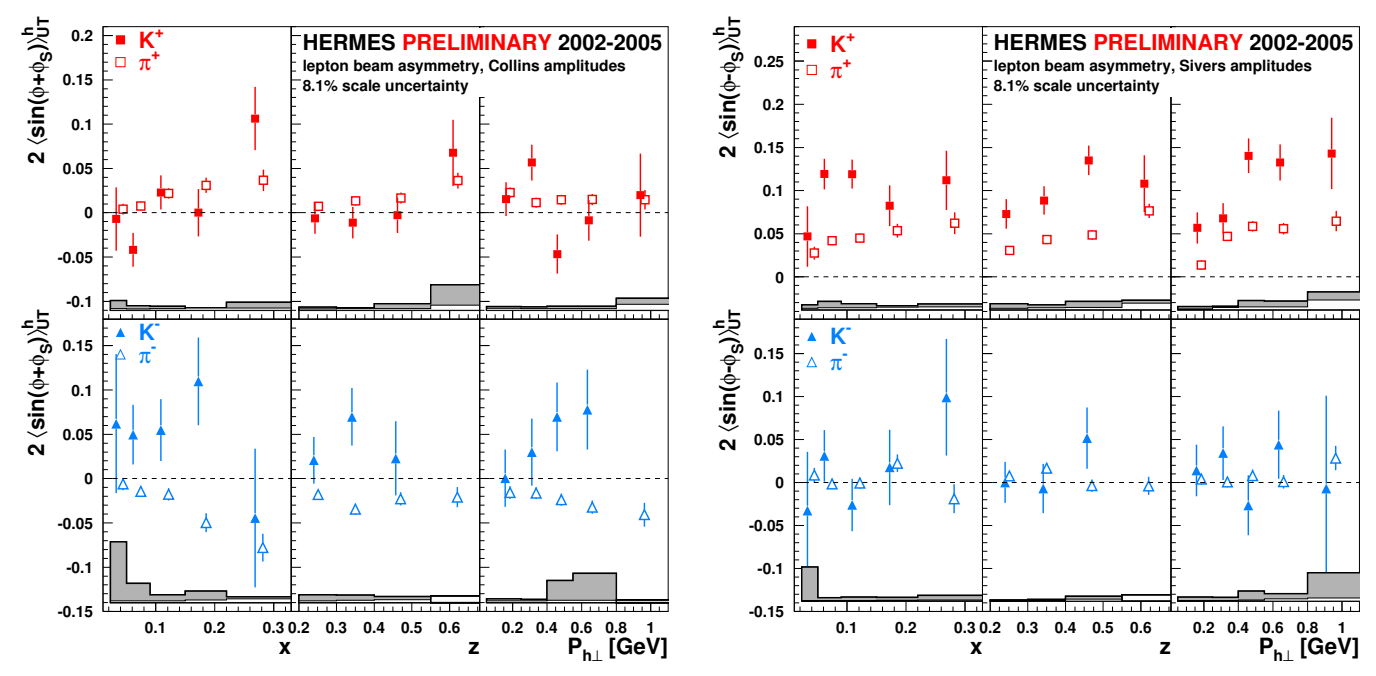

Figure 2: Collins amplitudes (left column) and Sivers amplitudes (right column) for charged kaons (closed symbols, as labelled) and charged pions (open symbols, as labelled) as function

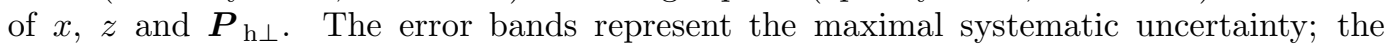
common overall $8.1 \%$ scaling uncertainty is due to the target polarisation uncertainty.

Here $P_{\alpha}^{z}$ denotes the degree of the target polarisation.

In Figures 1 and 2 the lepton-beam Collins and Sivers amplitudes as a function of $x$, $z$ and $\boldsymbol{P}_{\mathrm{h} \perp}$ are shown. Semi-inclusive DIS events were selected subject to the kinematic requirements $Q^{2}>1 \mathrm{GeV}^{2}, y<0.95, W^{2}>10 \mathrm{GeV}^{2}, 2 \mathrm{GeV}<\boldsymbol{P}_{\mathrm{h}}<15 \mathrm{GeV}, 0.2<z<0.7$ and $\theta_{\gamma^{*} \mathrm{~h}}>0.02 \mathrm{rad}$, where $\theta_{\gamma^{*} \mathrm{~h}}$ is the angle between the direction of the virtual photon and the hadron. The selected ranges in $x$ and $\boldsymbol{P}_{\mathrm{h} \perp}$ are $0.023<x<0.4$ and $0.05 \mathrm{GeV}<\boldsymbol{P}_{\mathrm{h} \perp}<2 \mathrm{GeV}$. These preliminary results are based on ten times more statistics than that in the publication [2] and are consistent with the published result.

The Collins amplitude is positive for $\pi^{+}$, compatible with zero for $\pi^{0}$ and negative for $\pi^{-}$. Also, the magnitude of the $\pi^{-}$amplitude appears to be comparable or larger than the one for $\pi^{+}$. This leads to the conclusion that the disfavoured Collins fragmentation function has a substantial magnitude with an opposite sign compared to the favoured Collins fragmentation function. For charged kaons no statistically significant non-zero Collins amplitudes are found. However, the Collins amplitudes for $K^{+}$are within statistical accuracy consistent to the $\pi^{+}$amplitudes.

The significantly positive average Sivers amplitudes observed for $\pi^{+}, \pi^{0}$ and $K^{+}$imply a non-vanishing orbital angular momentum of the quarks inside the nucleon. As the magnitude of the $K^{+}$amplitude is the larger than the one for $\pi^{+}$, the sea quark contribution to the Sivers mechanism appears to be important. Thus the orbital angular momentum of anti-quarks could be significant and highly flavour dependent. For $\pi^{-}$and $K^{-}$the Sivers amplitudes are consistent with zero.

Isospin symmetry of $\pi$-mesons is fulfilled for the extracted Collins and Sivers amplitudes.

Although formally, the contribution to the SSA of $\pi$-mesons and $K$-mesons from the decay of exclusively produced vector mesons is a part of the semi-inclusive DIS cross section, 

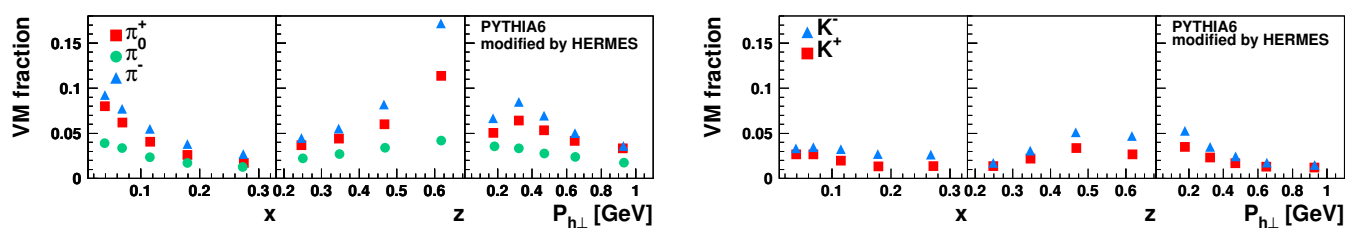

Figure 3: Simulated fraction of $\pi$-mesons (left column) and charged $K$-mesons originating from diffractive vector meson production and decay.

a too large contribution might contradict the assumptions of factorisation, i.e. summation over a larger number of contributing channels. As an indication, the simulated fraction of $\pi$-mesons and charged $K$-mesons originating from diffractive vector meson production and decay is shown in Figure 3

\section{Acknowledgements}

This work has been supported by the German Bundesministerium für Bildung und Forschung (BMBF) (contract nr. 06 ER 125I and 06 ER 143) and the European Community-Research Infrastructure Activity under the FP6 "Structuring the European Research Area" program (HadronPhysics I3, contract nr. RII3-CT-2004-506078).

\section{Bibliography}

\section{References}

[1] Slides: http://indico.cern.ch/contributionDisplay.py?contribId=150\&sessionId=4\&conf Id=9499.

[2] A. Airapetian et al. Single-spin asymmetries in semi-inclusive deep-inelastic scattering on a transversely polarized hydrogen target. Physical Review Letters, 94:012002, 2005.

[3] John C. Collins. Fragmentation of transversely polarized quarks probed in transverse momentum distributions. Nuclear Physics, B396:161-182, 1993.

[4] Dennis W. Sivers. Single spin production asymmetries from the hard scatterung of point- like constituents. Physical Review, D41:83, 1990.

[5] A. Airapetian et al. Quark helicity distributions in the nucleon for up, down, and strange quarks from semi-inclusive deep-inelastic scattering. Physical Review, D71:012003, 2005.

[6] John P. Ralston and Davison E. Soper. Production of dimuons from high-energy polarized proton proton collisions. Nuclear Physics, B152:109, 1979.

[7] Xavier Artru and Mustapha Mekhfi. Transversely polarized parton densities, their evolution and their measurement. Z. Phys., C45:669, 1990.

[8] Rober L. Jaffe and Xiang-Dong Ji. Chiral odd parton distributions and polarized drell-yan. Physical Review Letters, 67:552-555, 1991.

[9] J. L. Cortes, B. Pire, and J. P. Ralston. Measuring the transverse polarization of quarks in the proton. Z. Phys., C55:409-416, 1992.

[10] Matthias Burkardt. Quark correlations and single spin asymmetries. Physical Review, D69:057501, 2004.

[11] Stanley J. Brodsky, Dae Sung Hwang, and Ivan Schmidt. Final-state interactions and single-spin asymmetries in semi-inclusive deep inelastic scattering. Physical Letters, B530:99-107, 2002.

[12] Daniel Boer and P. J. Mulders. Time-reversal odd distribution functions in leptoproduction. Physical Review, D57:5780-5786, 1998. 